\title{
ENTRE LA NECESIDAD Y LA FE. LA PARTERA TRADICIONAL EN EL VALLE DEL RÍO CIMITARRA
}

\section{BETWEEN THE NECESSITY AND THE FAITH. THE TRADITIONAL MIDWIVES OF THE VALLEY OF THE CIMITARRA RIVER}

\author{
Celmira Laza VÁSQuez \\ Carmen Helena Ruiz de CÁrdenas ${ }^{*}$
}

\begin{abstract}
RESUMEN
Este artículo pretende analizar las características de las parteras tradicionales en la región del Valle del río Cimitarra en el Magdalena Medio colombiano a la luz de su contexto ambiental. Así, se realiza un acercamiento al contexto de la apartada región rural y de las características de las siete parteras tradicionales que participaron en el estudio. Lo anterior, asumiendo la postura teórica de Leininger de comprender las creencias y prácticas culturales inmersas en un contexto ambiental particular. Se evidencia cómo las características de las parteras tradicionales son el resultado de su adverso contexto social, económico y político de esta compleja región; y así mismo, cómo la necesidad de cuidar la vida determinó las razones que las impulsaron a hacer de la partería parte de su quehacer, sustentándolo en la fe a diferentes divinidades.
\end{abstract}

Palabras clave: Parteras tradicionales, prácticas de cuidado, creencias, contexto, aprendizaje, enfermería.

\begin{abstract}
This article analyzes the characteristics of traditional midwives in the Valley of Cimitarra river of colombian Magdalena-Medio region in relation to their environmental context. This approach was used to describe the situation on the remote rural region, and the essential features of the seven midwives who participated in the study. This study assumes the Leininger's theoretical viewpoint to understand the cultural beliefs and practices embedded in particular ambiance. This article support how the characteristics of traditional midwives are the result of its adverse social, economic and political adverse context within this complex region, and likewise, how the need to take care of life determined the reasons that led the midwifery to become part of their duty, supporting it in faith to different deities.
\end{abstract}

Key words: Traditional midwives, care practices, beliefs, context, learning, nursing.

Fecha recepción: 13/07/09 Fecha aceptación: 24/02/10

\section{INTRODUCCIÓN}

El quehacer de la partera tradicional en América Latina, históricamente, ha sido muy cuestionado por el sistema occidental así como por parte de los profesionales sanitarios. A este agente tradicional se le res- ponsabiliza, en parte, de la alta morbilidad y mortalidad materna en el continente, sin embargo, persiste un vacío en el conocimiento, las creencias y las prácticas que realizan las parteras al respecto.

Se encuentran trabajos en los que se hacen referencia a los problemas o complicaciones

* Enfermera. Investigadora docente Centro de Investigación y Desarrollo, Fundación Universitaria del Área Andina. BogotáColombia. E-mail: celmira.laza@gmail.com

** Enfermera. Profesora asociada Facultad de Enfermería, Universidad Nacional de Colombia. E-mail. ccruizd@unal.edu.co 
que se presentan en la madre y el recién nacido cuando el parto es atendido por una partera tradicional. Se refieren a que estos problemas están relacionados con el insuficiente conocimiento y las prácticas inadecuadas que aún persisten en la atención del parto por parte de estos agentes tradicionales. Además, que los riesgos se aumentan por las inadecuadas condiciones de las viviendas donde se atiende el parto y la falta de elementos necesarios para la atención $(1,2)$.

Sin embargo, en algunas regiones de América Latina, la existencia y actuar de estos agentes se debe a diferentes razones como las de tipo históricas, ya que siempre las parteras tradicionales han jugado un papel central en la atención y cuidados a las mujeres en los momentos del embarazo, parto y puerperio. Su histórico quehacer se ha mantenido como una forma de preservar la vida humana, y en la actualidad se consolida frente a los problemas de inaccesibilidad a los servicios de salud, tanto por razones estructurales propias de éstos como por cuestiones culturales, convirtiéndose la medicina tradicional y doméstica en las regiones apartadas, aisladas o marginadas, la única opción para la atención de la salud de amplios sectores de la población (3).

En el contexto rural colombiano, caracterizado en la actualidad por el desarrollo del conflicto social y armado, abandono estatal y la ausencia de una política de inversión social coherente con la realidad actual, la partera tradicional se consolida, ya que por estas condiciones se convierte en la única opción para la atención de la mujer y el recién nacido durante el parto. A pesar de esto, en Colombia persiste el vacío y una cierta ignorancia sobre los contextos en que se desarrolla el trabajo de la partera, sus características, las creencias y las prácticas que llevan a cabo con las parturientas. Otro elemento encontrado en la literatura científica es el rechazo de las mujeres gestantes de esta región rural a ser atendidas por los profesionales de la salud y en los servicios formales, lo cual no es más que la expresión del enfrentamiento cultural de las mujeres frente a los cuidados incoherentes con su cultura y el modo de vida de éstas; así como la forma en que se concibe y se cuida, según la cultura, esta etapa de la vida de la mujer.

Así, la gestación, el parto y el puerperio son considerados como eventos propios e íntimos de la vida de la mujer que involucra el pudor de ésta. Por esto, en el caso del parto, el ambiente del hogar propio o de la partera forma parte de la intimidad, la solidaridad y la calidez necesarias para que el parto salga bien. A pesar de la problemática para el acceso de las mujeres a los servicios en salud de la región, la preferencia por el cuidado de la partera durante el parto se relaciona directamente con los problemas de acceso cultural a los servicios de salud; con lo cuales, las mujeres expresaron su desacuerdo por la infraestructura fría, poco cómoda, la imposibilidad de estar acompañada por su familia y el trato de los profesionales de la salud (4).

Este manuscrito pretende analizar las características de las parteras tradicionales a la luz de su contexto ambiental, a partir de los resultados parciales de un trabajo investigativo de mayor alcance que describió los cuidados que brindan las parteras tradicionales a la mujer y el recién nacido durante la atención del parto, a partir de las creencias y prácticas, en la apartada región rural del Valle del río Cimitarra en el Magdalena Medio colombiano realizado durante el 2007 y 2008. La investigación se desarrolló en varias veredas de la zona rural y la ciudad de Barrancabermeja y participaron siete parteras que gozaban de gran reconocimiento por parte de sus comunidades. De corte cualitativo, se realizó una etnografía focalizada o minietnografía. La información se obtuvo mediante la observación participante y entrevistas cualitativas a profundidad que fueron analizadas con la técnica de Spradley. En el presente manuscrito se describen las características de las participantes y del contexto en el cual viven y desarrollan su valioso quehacer. El trabajo 
se estructuró sobre la propuesta teórica de Madelein Leininger de "La diversidad y universalidad del cuidado cultural" (5).

\section{El complejo contexto ambiental del Valle del río Cimitarra en el Magdalena Medio}

La teoría de "La diversidad y universalidad del cuidado cultural" se enfoca en descubrir las diferencias y universalidades del cuidado humano para generar un nuevo conocimiento que guíe las prácticas del cuidado de enfermería, con el fin de proporcionar un cuidado profesional culturalmente congruente y competente que le llevará al cliente $\mathrm{y}$ al grupo salud y bienestar (5).

Por esto, uno de los elementos fundamentales a tener en cuenta para la descripción de los cuidados y prácticas culturales son las dimensiones de la estructura cultural y social, que hace alusión a los patrones dinámicos y rasgos o a los factores interrelacionados estructurales y organizacionales de una cultura particular (subcultura o sociedad) la cual incluye valores religiosos, familiares (sociales), políticos (y legales), económicos, educativos, tecnológicos y culturales, factores etnohistóricos y la forma en que estos valores pueden estar interrelacionados y funcionan para influenciar el comportamiento humano en los diferentes contextos ambientales (5). A su vez, el contexto ambiental es la totalidad de un evento, situación o experiencia particular que le da significado a las expresiones humanas, interpretaciones e interacciones sociales en ambientes particulares físicos, ecológicos, sociopolíticos y/o culturales (5). Por esto, para comprender las características, cuidados, prácticas y creencias de las parteras tradicionales, es necesario analizar y comprender el contexto en el cual desarrollan su quehacer.

El Valle del río Cimitarra es una región inmersa en el Magdalena Medio colombiano, en la franja occidental del río Magdalena, entre los departamentos de Antioquia y Bolívar. Se forma en el trayecto que hace este río en búsqueda del río Magdalena, y que recorre zonas rurales de los municipios de Remedios y Yondó, en Antioquia; y Cantagallo y San Pablo, en el sur de Bolívar, en Bolívar donde finalmente desemboca (6).

La historia de esta región ha estado marcada por el poblamiento que se originó por procesos de colonización a causa de las migraciones generadas por persecuciones políticas y sociales en las décadas de 1950, 1960 y 1980. En ésta última, miles de campesinos buscaban refugio huyendo del paramilitarismo, que para entonces se consolidaba en Puerto Boyacá, Puerto Berrío y otras zonas cercanas (7). Por estas migraciones, su población es diversa y la región se conformó por campesinos desplazados forzadamente en repetidas ocasiones; entremezclándose acervos culturales de antioqueños, bolivarenses, santandereanos, entre otros, que conviven y han hecho de esta región su hogar.

En los años ochenta, los campesinos de esta vasta región se organizaron en cooperativas, buscando un modo de subsistencia económica y a su vez como forma de organización social. El abandono estatal y el constante señalamiento y asesinato de sus pobladores fue determinante para la construcción y actual consolidación de una cultura organizativa basada en los principios de subsistencia e intercambio solidario. Su primera manifestación fue la Coordinadora Campesina del Magdalena Medio para intentar canalizar esa fuerza y capacidad de organización de los pobladores en el momento en que empezaba a consolidarse el paramilitarismo en la región. En los noventa se constituye la Asociación Campesina del Valle del río CimitarraACVC con los mismos objetivos (7).

Esta zona rural presenta los mismos problemas y conflictos que padece la región en su totalidad: abandono estatal, inexistencia de servicios como salud, educación, vivienda, servicios públicos domiciliarios, una economía extractiva, mal estado de las vías, concentración de la tierra y presencia de cultivos de uso ilícito y desarrollo del conflicto 
social y armado. "En el Magdalena Medio, las necesidades básicas insatisfechas son de un $80 \%$ (con tendencia a crecer). Por otra parte, los derechos humanos son violados recurrentemente, a causa de la implantación de un modelo de desarrollo autoritario, que sólo garantiza el libre mercado. Este hecho ha generado la intensificación de las acciones contrainsurgentes y de control territorial, para incorporar estas zonas al modelo neoliberal. En estas condiciones, una propuesta de resistencia es, en un principio, un impulso de vida para los pobladores. Una ilusión que, al presentarse como colectiva, va tomando fuerza y va adquiriendo forma propia (7)".

Pese al desolador panorama que ha marcado a estas comunidades rurales, en esta región se han estructurado propuestas alternativas de modelos de desarrollo, y las han defendido como posibilidad real de un nuevo sistema de relaciones económicas, políticas y sociales; concretado en la organización comunitaria y el desarrollo de un importante proceso de resistencia pacífica desde hace más de veinte años. Este proceso ha sido liderado en los últimos doce años por la (ACVC $)^{1}$, alcanzándose una población con un alto grado de organización y formación política. En la actualidad existe una economía y organización de tipo solidaria y con acciones enmarcadas en la construcción de autonomía alimentaria.

\section{¿Quiénes son, de dónde son, de dónde vie- nen las parteras tradicionales del Valle del río Cimitarra?}

En Colombia la partería es ejercida en forma exclusiva por mujeres, cuyo promedio de edad supera los 55 años y quienes han permanecido conviviendo durante mucho tiempo en la comunidad y por tanto tienen un conocimiento muy familiar de la misma. $\mathrm{Su}$ proceso de aprendizaje es informal, la mayoría adquiere los conocimientos necesarios para su trabajo a raíz de experiencias propias o circunstancias accidentales que las forzaron a atender un parto; esto ocurre a la edad de 19 años promedio. Lo predominante en su aprendizaje es la observación y la práctica. Su conocimiento se enriquece durante muchos años de práctica empírica (8).

Las parteras tradicionales presentan unas características específicas dentro del abanico de agentes sanitarios tradicionales. Así, ellas nacen como líderes de sus comunidades y por esto, ejercen gran influencia sobre las prácticas de salud de éstas (2); son parte de las comunidades, teniendo una familiaridad con la vida cotidiana de los colectivos y en especial, con las mujeres; por lo cual tiene pocas limitaciones para abordar temas femeninos (9); tienen concepciones sobre el ser humano y la naturaleza diferentes al saber médico. Por esto, recurren a la práctica de la medicina tradicional y su conocimiento es producto de un saber tradicional que involucra la tradición oral y el aprendizaje empírico y práctica (1). Su trabajo en las comunidades se desarrolla en torno de una economía de subsistencia para las familias y de los colectivos (10).

Las siete parteras tradicionales del Valle del río Cimitarra que participaron en el estudio se encontraban en un rango de edad de los 46 y 66 años. La mayoría eran de zonas distintas al Valle del río Cimitarra. Todas eran de origen campesino y sobrevivían gracias a las actividades propias del campo. $\mathrm{Su}$ quehacer lo desarrollaban en una sociedad enmarcada en los valores del proceso de resistencia pacífica y estructurado sobre una economía de subsistencia y solidaria. Por esto, todas participaban activamente en

\footnotetext{
${ }^{1}$ La ACVC es una organización social no gubernamental campesina de carácter regional que desarrolla un trabajo organizativo, político y social. Dentro de los objetivos organizacionales se encuentran los de resolver los desequilibrios sociales existentes como una de las bases para el logro de la paz y propender por el derecho de una vida digna y la defensa de los derechos humanos en la región.
} 
los procesos organizativos como las Juntas de acción comunal y en el proceso de base de la ACVC, siendo el reconocimiento de su trabajo de tipo moral y no económico. Así lo expresó una de ellas:

"Bueno, a veces me pagan y otros no. No hay problemas sino me paga, las necesidades dice Dios sino hasta luego. Sino tienen, no es problema. Uno lo hace porque toca, porque me gusta y quisiera saber más. Cuando me pagan, es porque la gente quiere y tiene con que...o es que a veces la gente no tiene ni con qué cortar el bebé, ni con qué envolver al bebé". Partera tradicional de 65 años.

Residían en diferentes veredas de la región rural desde hacía entre 6 y 10 años. Llegaron desplazadas forzadamente producto del conflicto social y armado, sufriendo en carne propia las consecuencias de este fenómeno que se ha convertido en una crisis humanitaria de dimensiones impensables en Colombia:

“...En el 80, cuando empezaron las matanzas de los paramilitares, de ahí pa acá yo he andado mucho. En la única parte donde me he establecido un poco, es acá... Cuando esas matanzas que principiaron de los paramilitares, yo vivía en el departamento de Santander. Bueno, y ahí me fui, me fui para los Llanos. En los Llanos alcance a vivir 18 años, de ahí me toco venirme botando lo que tenía, eso fue... en el 97, 98. Ellos entraron a matar gente. Una vez, hicieron una reunión los paramilitares y nos dijeron: que el que tuviera esta tierra y fuera viejo... directamente, sino se quería morir, se podía ir. Entonces, yo tenía 18 años allá y era vieja y entonces me toco venir de allá, dejar allá la finca y venirme con mi marido, el finao con 4 hijas y andar como alma que lleva al diablo". Partera tradicional de 66 años.

\section{La necesidad frente a la adversidad: forja- doras de las parteras tradicionales}

La necesidad de cuidar la vida, representada en la parturienta y su hijo por nacer, frente a la ausencia de profesionales que asumieran estas funciones, son las razones que llevaron a estas mujeres a convertirse en parteras tradicionales. En todos los relatos emergen tres de éstas:

La necesidad de contar con mujeres que ayudaran o "acompañaran" a las parturientas el parto, es quizás la razón mas significativa, ya que históricamente en esta región no se ha brindado una atención sanitaria regular por parte del sistema de salud formal. Así lo narra una de ellas:

"De ahí, de unos 38 años empecé a partear las mujeres que me pedían el favor, porque yo nunca tuve cursos, nunca hice cursos de enfermería o alguna cosa, sino para ayudarle a las mujeres de parto, me invitaban a que las acompañara, que las ayudaba porque ellas estaban solas y no tenían quien las ayudara". Partera tradicional de 48 años.

La segunda, es la importancia que en este contexto se le otorgan a la maternidad y al futuro. Con respecto a esto, una de ellas expresó:

“Traer un niño al mundo? Qué le digo yo a usted? Una bendición porque es una vida más para el mundo. La niñez es el futuro para el día de mañana. Uno sobre todo se debe cuidar a los niños y toda mujer que tenga el privilegio de ser madre, muy lindo, porque es una bendición de Dios". Partera tradicional de 65 años.

La tercera, los choques culturales que han tenido y que se han vuelto parte de las experiencias y recuerdos negativos en los encuentros con los servicios y agentes sanitarios formales. Esto es resultado del desconocimiento del sistema formal sobre la realidad social y las prácticas y creencias culturales de estas comunidades rurales. El siguiente es uno de los recuerdos negativos:

"Una muchacha de aquí me dijo que no quería ir al hospital porque ahí a veces que 
están pujando y no las atienden ligero o se descuidan con tantas personas. Muchas veces, habido el caso que la mujer va a orinar y nacen dentro de los baños. O si una mujer, de las que son flojas y son gritonas o cierran los pies cuando va a parir, las rajan y les hacen cesárea”. Partera tradicional de 65 años.

Las formas de aprendizaje eran diferentes a las establecidas por el sistema biomédico. Esto es lógico si se tiene en cuenta las escasas posibilidades de estudio que tienen los pobladores de esta región, tanto por circunstancias económicas como por la escasa presencia del sistema educativo. De hecho, la mayoría apenas sabían leer y escribir y una de ellas se consideró analfabeta. Así, a pesar del amplio conocimiento en el arte de la partería, ninguna de las informantes fue capacitada por el sistema sanitario formal, aunque algunas realizaron cursos de enfermería y capacitaciones en primeros auxilios por parte de la Cruz Roja colombiana y otras organizaciones no gubernamentales.

Las formas de aprendizaje han sido variadas. La más significativa fue mediante la información recibida y observación del quehacer durante la atención del parto de otras mujeres de la familia, entre las que destacan la madre y la suegra:

"No, yo viendo, por ejemplo una amiga que se enfermaba o algo así, y me convidaba a acompañar y yo estaba mirando, hasta que se resuelve uno. Nunca me enseñaron...”. Partera tradicional de 61 años.

"En el Urabá, entre nosotras mismas, como el cuento. Porque yo, ah entonces y cuando ella se enfermaba, ella me mandaba a llamar y como yo veía a la suegra mía que era partera, ah entonces yo la veía y ella me decía: cuando usted valla a partear, haga así y así. Entonces, cuando me invitaban a que acompañara a una señora yo miraba como le hacían al ombligo y eso...y lo que veía de mi suegra y yo hacía lo mismo". Partera tradicional de 58 años.
El método del ensayo-error como forma de aprendizaje y validación de su práctica, así cómo las situaciones vividas durante sus gestaciones y partos. Por ejemplo, una de ellas comenta cómo a partir de las actividades que realizaba cuando estaba de parto, lo hace con las parturientas:

"Con la primera, yo misma me puse a pensar y dije: bueno, yo cuando estoy de parto, yo camino pa allá, pa acá, en la casa. Le doy la vuelta a la casa o en la cocina, entonces yo sé, tomo el ejemplo de mí de que una mujer no puede dejarse acostar porque entonces el pelao se demora en nacer". Partera tradicional de 48 años.

El aprendizaje por los accidentes de la vida también es llamativo. "Yo, yo aprendí con ella (señala a la hija). Porque era que nosotros estábamos solos en una montaña. Solos no porque no habían casas pero entonces estaban muy lejos (...) Y vea, que no soy partera pero me tocó con ella. Y ahí fue cuando me tocó, que mejor dicho... ay, señor bendito!". Partera tradicional de 59 años.

Otros aspectos que se resaltaban en el aprendizaje de las parteras tradicionales fueron:

- Una estrecha relación de éstas con otros agentes tradicionales de la región como los componedores de huesos, los hierbateros y los rezadores. Con éstos ha existido un intercambio continuo de saberes, lo que ha aumentado su cúmulo de conocimientos.

- El profundo conocimiento y utilización en sus prácticas de las diferentes plantas de la región, las cuales han clasificado por sus características de ser frías o calientes.

- La forma de transmisión del conocimiento ha sido de tipo oral.

- La superación y cualificación continua ha sido mediante la práctica, la observación y los consejos de otras colegas de mayor edad o experiencia. 


\section{La fe: el soporte de la práctica}

La amplia experiencia de la práctica de las parteras tradicionales del Valle del río Cimitarra se sustentaba en la fe. De confesión católica, antes de atender a una parturienta, se encomiendan a Dios y a la Virgen del Carmen, confiándoles su actuar y la vida de la parturienta y el recién nacido:

"Yo les decía: yo no soy partera. Yo no tengo título ni cartón de partera. Yo simplemente, si veo que la situación o que la mujer está muy apurada, que no puede salir, que porque no tiene plata, yo los ayudo. Pero si tiene fe, pongamos fe en Él. Pues con la fe en mi diosito usted se va alentar. Después de Dios no hay más nada". Partera tradicional de 48 años.

Todas las parteras tradicionales se encomendaban a la Virgen del Carmen. Un ejemplo de esto es la oración que a ella le han dedicado:

"Virgencita del Carmen, en tus manos queda, que nos ayude, en tus manos queda con tú poder. Virgen del Carmen, que sea un parto feliz, que no haya novedad en el parto ni ella ni su criatura, que se aliente ligero". Partera tradicional de 59 años.

La devoción a esta Virgen en la región del Magdalena Medio se remonta a la época de la colonia y se reafirma en el siglo XX con la evangelización de la Compañía de Jesús, forjando así la devoción en Ella. Su historia, en el marco de un contexto de conflicto social y armado y desplazamientos forzados, también dan cuenta de su carácter protector, referido por muchos habitantes, los cuales le atribuye la ayuda prestada durante los destierros y para escapar de la zona de guerra en medio de combates o ataques de los actores armados (11).

En el caso de la devoción de las parteras tradicionales, "La fe en la Virgen del Carmen, no se puede separar con facilidad de la fe en el Divino niño, ya que se trata de una señora con un niño". En esta fe se expresa todo el saber tradicional, acumulado en el trabajo con hierbas y el saber de las parteras, devotas todas de esta especial Virgen, referencia central de las madres y la crianza (11)". "Uno es devoto de la virgen del Carmen, anjá, porque la virgen es devoto de la mujer, porque ella también tiene un niño y sufrió como uno. Uno pone el cuadro de la virgen, le pone una vela completa y si uno se la pone y uno le pide que si antes de que se termine la vela, la mujer haya tenido y eso pasa". Partera tradicional de 48 años.

\section{A manera de conclusión... el influyente contexto social y cultural}

Leininger, al desarrollar su teoría, se planteó ¿De qué manera influía y contribuía el contexto ambiental y los factores sociales, económicos y los valores culturales en los patrones de salud, el bienestar y las prácticas de cuidado? El desarrollo de la teoría confirmó que sí influyen. En este sentido señaló que dentro de la evaluación del cuidado cultural es necesario no sólo estudiar las creencias, significados, valores y prácticas culturales, sino además, la visión general, el contexto medioambiental, la etnohistoria y la estructura de los valores sociales. Todo lo anterior, con el propósito de tener una perspectiva holística en la descripción y comprensión del sistema de los cuidados genéricos de un pueblo (5).

Siguiendo sus postulados, es imposible acercarse a la descripción de los cuidados culturales de las parteras tradicionales sin la comprensión de su contexto social, político y económico. Por esto, sin lugar a equivocación, un elemento de gran trascendencia en la formación y consolidación de estas mujeres en el Valle del río Cimitarra es cómo el propio contexto ha sido un elemento determinante en su configuración y su permanencia a lo largo de la historia de esta región. Quizás, si las condiciones de vida fueran más favorables, su existencia no sería una realidad 
a gritos que los profesionales y los sistemas de salud desearan ignorar. En este sentido, se puede afirmar que su actuar es parte de la necesidad de sobrevivir de todo un pueblo. Lo anterior se evidencia en sus características $\mathrm{y}$ en las razones por las que decidieron convertirse en parteras tradicionales. En los dos aspectos hay una coincidencia con lo descrito en la literatura científica.

Como constructoras y forjadoras del pueblo del Valle del río Cimitarra, su aporte a la historia y a la identidad de esta región ha sido ser las guardianes de la nueva vida y de los secretos, cuidados y creencias entorno a la atención del parto, en un contexto marcado por la violencia, la guerra, el desplazamiento forzado y el abandono estatal.

En el marco del proceso de la resistencia pacífica de este pueblo campesino, su contribución va más allá de aportar a la continuidad de la vida y a la sobrevivencia de su pueblo, no sólo de la guerra, sino también del olvido. Así, más que hacer historia, el quehacer de las parteras tradicionales del Valle del río Cimitarra es la historia de este pueblo.

\section{REFERENCIAS}

1. Méndez R, Cervera M. Comparación de la atención del parto normal en los sistemas hospitalario y tradicional. Salud pública Méx. 2002; 44 (2):129-136.

2. Hincapié E, Valencia C. Capacitación de las parteras y su relación con la mortalidad perinatal del municipio de Quinchía, Colombia. Colombia med 2001; 31: 1115.

3. Campos-Navarro R. Prácticas médicas populares: algunas experiencias sobre el proceso de autoatención curativa. En: Campos-Navarro R, comp. La antropología médica en México. México, DF: Instituto Mora/Universidad Autónoma
Metropolitana, 1992:186-210.

4. Medina M. Salud sexual y reproductiva en el Magdalena Medio: caracterización socioantropológica. Bogotá: PDPMM FNUAP, 2001.

5. Leininger M. Transcultural Nursing Concepts, Theories, Research \& Practices. 2a ed. New York: McGraw-Hill, Inc. College Costom Series, 1995.

6. Asociación Campesina del Valle del río Cimitarra. La Zona de Reserva Campesina del Valle del río Cimitarra: una iniciativa agraria de paz. Agencia prensa rural 2008. Hallado en: http://www.prensarural.org/spip/spip.php?article1098 [Acceso el 10.03.2007].

7. Becerra S. La resistencia campesina en Colombia. Experiencia de la Asociación Campesina del Valle del río Cimitarra (ACVC). Agencia prensa rural 2005. Hallado en: http://www.prensarural.org/ acvc/becerra20050202.html [Acceso el 10.03.2007].

8. Eslava D. Los agentes tradicionales de salud: otras alternativas para las comunidades campesinas. El caso de Cundinamarca y Santander. Revista Investigación y Educación en Enfermería 1998; XVI (2): 57-71.

9. Valdez R, Arenas L, Hernández I. Experiencia de las parteras en la identificación de mujeres maltratadas durante el embarazo. Salud pública Méx 2004; 46 (1). Hallado en: http://scielo.unam.mx/scielo.php?script $=$ sci_arttext $\&$ pid $=$ S0036$36342004000100008 \& \operatorname{lng}=\mathrm{en} \& \mathrm{nrm}=$ iso [Acceso el 10.03.2007].

10. Linares M, Moral I, Medina E, Contreras S. Inventario etnográfico de las parteras de Sierra Mágina. Aproximación a su relación con las matronas. Index Enferm 2005; 14 (51):10-14.

11. Solarte R. Voces y silencios: Paradojas de la Virgen. Revista Teología Xaveriana 2004; 149: 98-102. 